\title{
ORIGINAL
}

\section{DISTRIBUCIÓN ESPACIAL DE LA TUBERCULOSIS EN ESPAÑA MEDIANTE MÉTODOS GEOESTADÍSTICOS}

\author{
Diana Gómez-Barroso (1,2), Elena Rodriguez Valín (2,1), Victor Flores Segovia (2), Rebeca Ramis \\ Prieto (2,1), Jose Luís del Barrio Fernández (3), Fernando Simón Soria $(2,1)$.
}

(1) CIBER en Epidemiología y Salud Pública - CIBERESP.

(2) Centro Nacional de Epidemiología, Instituto de Salud Carlos III.

(3) Facultad de Ciencias de la Salud Universidad Rey Juan Carlos. Departamento de Medicina Preventiva, Salud Pública, Inmunología y Microbiología Médica.

\section{RESUMEN}

Fundamentos: La incidencia de tuberculosis (TB) se ha asociado a factores, epidemiológicos y sociales. En España, la TB es una enfermedad de declaración obligatoria e individualizada. Las tasas de TB respiratoria experimentan un descenso constante en los últimos años. El objetivo es valorar la asociación entre la morbilidad por TB respiratoria y variables socioeconómicas y epidemiológicas así como su distribución espacial mediante métodos geoestadísticos.

Método: Las tasas de incidencia se estandarizaron por edad y sexo con datos de la Red Nacional de Vigilancia (2006). Las variables socioeconómicas incluidas son: condición socioeconómica, nivel de estudios, tasa de hacinamiento, densidad de población, tasa de inmigración estandarizada por sexo, tasa de analfabetismo, tasa de paro, gasto medio en euros por persona. Las variables epidemiológicas incluidas han sido la tasa de SIDA y la tasa de incidencia de gripe. Se realizó un análisis multivariable mediante un Modelo Lineal Generalizado poisson. Se aplicó la técnica geoestadística Cokringing ajustada por las variables estadísticamente significativas para ver la distribución espacial de riesgo.

Resultados: Las variables estadísticamente significativas son la tasa de hacinamiento, tasa de inmigración, tasa de analfabetismo, tasa de paro, gasto medio euros por persona, tasa de gripe y tasa de sida. La técnica geoestadística muestra una variabilidad espacial del riesgo y una concentración del riesgo en el noroeste y sureste de la península.

Conclusiones: Los resultados permiten afirmar que el método Cokriging es una herramienta útil para representar la distribución espacial del riesgo. Existe asociación entre variables socioeconómicas, epidemiológicas y TB en España.

Palabras clave: Tuberculosis. Análisis espacial. Análisis multivariable.

Correspondencia:

Diana Gómez-Barroso

Instituto de Salud Carlos III

Monforte de Lemos n 5, pabellón 12. 28071 Madrid

Correo electrónico: dgomez@isciii.es

\section{ABSTRACT \\ Space Distribution of Tuberculosis in Spain by Geostatistical Methods}

Background: Tuberculosis incidence has been associated with many factors, both epidemiological and social. In Spain, tuberculosis is a statutorily notifiable disease requiring individualised reporting. During the last few years rates of respiratory tuberculosis show a steady decline. This study sought to assess respiratory tuberculosis morbidity and mortality in association to socio-economic and epidemiological covariates and estimate its spatial distribution across the country, using geostatistical methods.

Methods: Respiratory tuberculosis incidence rates were standardised by age and sex with the data of the National Epidemiological Surveillance Network (RENAVE, Red Nacional de Vigilancia Epidemiológica) for 2006. The following socio-economic variables were included in the study: socio-economic status, educational level, overcrowding rate, population density, standardised immigration rate by sex, unemployment rate and average spending per person in euros. The epidemiological variables included were, such as, AIDS rate and the influenza incidence rate. To assess the association of covariables a multivariate analysis was performed using a Generalised Linear Model assuming Poisson distribution. The goestatistical method Co-kriging was adjusted with the significant variables to built the spatial distribution of risk.

Results: The statistically significant covariates were overcrowding rate, standardised immigration rate by sex, educational level, unemployment rate, average spending per person in euros, AIDS rate and the influenza incidence rate. The geostatistical method shows spatial variability of the risk with higher risks in the northwest and southeast of the peninsula.

Conclusion: Results prove that the Co-kriging method is a useful tool to show the spatial distribution of risk. Alternatively, tuberculosis is associated with both social and epidemiological covariates.

Key words: Tuberculosis. Spatial analysis. Multilevel Analysis.

(*) En este trabajo no existen conflictos de intereses. 


\section{INTRODUCCIÓN}

La tuberculosis (TB) sigue representando un problema de Salud Pública a nivel mundial. Según la Organización Mundial de la Salud (OMS), el número estimado de nuevos casos en 2006 fue de 9,2 millones, lo que supone un $0,6 \%$ de aumento respecto al año $2005^{1}$. Según el último informe de la Red Europea de Vigilancia de Tuberculosis (EuroTB) ${ }^{2}$, en la Región Europea de la OMS se notificaron 422.830 casos de tuberculosis en 2006. Aunque la tendencia en el número de casos para el total de la Región es descendente, existen grandes variaciones según regiones. En Europa occidental y en los Balcanes la tendencia en el periodo 2002-2006 fue descendente, mientras que en el Este fue ascendente.

La incidencia de tuberculosis se ha asociado a muchos factores, tanto epidemiológicos como sociales. Los datos disponibles señalan que mayoritariamente, la tuberculosis es una enfermedad de hombres, aunque estas diferencias no pueden ser explicadas solamente por el hecho biológico, sino que también intervienen otros factores como exposición a distintos factores de riesgo, entre los que se incluyen estilos de vida, tabaco, y ocupación ${ }^{3 ;}$. Se estima que más del $75 \%$ del aumento global estimado de la incidencia de tuberculosis es el resultado de factores demográficos como el crecimiento de la población ${ }^{1}$ y los cambios en la estructura por edad. En los países industrializados se señala que, aparte de la longevidad y el envejecimiento de su población, hay otros factores como la privación socioeconómica, la inmigración de países de alta incidencia, el declive de los programas de tuberculosis, la fármaco resistencia y la epidemia del virus de la inmunodeficiencia humana (VIH) y SIDA, han contribuido sustancialmente a las tendencias actuales de la incidencia $^{5-7}$.

En España, la tuberculosis es una enfermedad de declaración obligatoria e indivi- dualizada de acuerdo a características básicas como son la edad y sexo, además de otros datos de interés clínico y epidemiológico $^{8-11}$. La epidemiología de la tuberculosis en los años 80 y 90 estuvo muy influenciada por la epidemia de VIH, especialmente por los usuarios de drogas por vía parenteral ${ }^{12}$.

Desde que se dispone de datos individualizados a nivel nacional, las tasas de tuberculosis respiratoria en España han experimentado un descenso constante en el periodo 1999-2004 (22 casos/100.000 habitantes en 1999 hasta 16 en 2004, media de descenso anual 5\%), observándose desde 2004 una estabilización en la notificación. Las causas de esta estabilización no están claras, aunque los datos de vigilancia muestran en los últimos años un aumento del número de casos nacidos fuera de España, (en 2004 el número de casos de tuberculosis respiratoria nacidos fuera de España fue de 602, incrementándose en 2005 a 1.143 y en 2006 a 1.275$)^{13}$. Esta tendencia se corresponde a la observada en algunos países de Europa occidental, como Irlanda, Italia y Reino Unido ${ }^{2}$. La tasa bruta media de notificación de tuberculosis respiratoria en España fue de 15 casos/100.000 habitantes en 2006, existiendo diferencias entre las distintas regiones ${ }^{13}$; 14 . Estudios realizados en diferentes lugares del territorio nacional sugieren que hay una asociación entre factores sociales (hacinamiento, desempleo, no tener hogar) y riesgo de tuberculosis $6 ; 15$.

Diversos estudios en diferentes países han analizado la distribución espacial de la TB y la asociación con diferentes factores socioeconómicos ${ }^{16-21}$.

El objetivo de este trabajo es valorar la asociación existente entre la morbilidad por TB respiratoria y variables socioeconómicas y epidemiológicas así como su distribución espacial en el territorio mediante métodos geoestadísticos. 


\section{MATERIAL Y MÉTODOS}

Este es un estudio analítico en el que se ha realizado un análisis geoestadístico para ver la distribución de la morbilidad por TB respiratoria ajustada por factores socioeconómicos y epidemiológicos con el método Cokriging.

Las tasas de incidencia estandarizadas por edad (se ha considerado 4 grupos de edad, de 0 a 14 años, de 15 a 44, de 45 a 65 y mas de 65) y sexo de tuberculosis respiratoria han sido calculadas a partir de los casos declarados a la Red Nacional de Vigilancia Epidemiológica (RENAVE) en el año 2006 para cada provincia por 100.000 habitantes. Se han excluido del análisis aquellos casos en los que las variables de año o la provincia estaban incompletas o eran desconocidas. Se consideraron sólo los casos de tuberculosis respiratoria, definiéndose como tuberculosis respiratoria la que afecta al parénquima pulmonar y al árbol traqueobronquial, la tuberculosis laríngea, así como la pleural y de los ganglios linfáticos intratorácicos. Se incluyeron todos los casos declarados, estuvieran o no confirmados por laboratorio. Se han analizado las provincias peninsulares, excluyéndose del análisis los territorios insulares, y las ciudades autónomas de Ceuta y Melilla por no existir una correlación espacial con la península. Para la estandarización de las tasas se ha utilizado la población de referencia del padrón de 2006 del Instituto Nacional de Estadística (INE).

Las variables socioeconómicas incluidas en el estudio provienen de diferentes fuentes de información así, del censo 2001 publicado por el INE se ha extraído la condición socioeconómica media por hogar a nivel provincial (variable proporcionada por el INE que se obtiene combinando la información de las variables de ocupación, actividad y situación profesional), nivel de estudios y la tasa de hacinamiento (superficie útil por numero de miembros de la vivienda). Del Padrón 2006 publicado por el INE se han utilizado la densidad de población (habitantes por $\mathrm{Km}^{2}$ ), y la tasa de inmigración estandarizada por sexo. De la encuesta de Indicadores Sociales publicada por el INE en 2006 se han considerado la tasa de analfabetismo, tasa de paro y el gasto medio en euros por personas considerándolo como una variable de situación económica. Las variables epidemiológicas incluidas han sido: tasa de SIDA (tasa por 100.000 habitantes del Registro Nacional de SIDA, datos actualizados a diciembre del 2007) y la tasa de incidencia de gripe (calculada a partir de los casos de declarados al Sistema de Vigilancia de la Gripe en España en el año 2006).

Se calculó el centroide de cada provincia para agregar los casos a nivel provincial y se realizó el análisis de correlación espacial entre las variables de estudio.

En el análisis de correlación se valoraron las cuatro direcciones cardinales principales y las tendencias de distribución espacial de los datos.

Se realizo un análisis multivariable mediante un Modelo Lineal Generalizado (GLM) ${ }^{22}$ para las tasas de incidencia. En todos los casos, asumimos una distribución de Poisson. Aquellas variables no significativas o que presentaron colinearidad fueron excluidas del modelo para el posterior análisis espacial.

Se realizó la estimación del riesgo de TB para el resto del territorio peninsular español para el año 2006 ajustado por las variables significativas utilizando el método multivariable Cokriging ordinario mediante la función del semivariograma cruzado.

Para ajustar las variables del semivariograma cruzado se valoraron tres modelos: el exponencial, el esférico y el gaussiano aplicando el que mejor se ajustaba a las variables estudiadas. Para ajustar los parámetros 
se utilizo un modelo lineal de corregionalización ${ }^{23 ;} 24$ que establece que los semivariogramas individuales y el cruzado sean combinaciones lineales de modelos de semivariogramas. Para la estimación del semivariograma cruzado ${ }^{17 ; 25}$ se utilizó esta formula:

$\gamma_{Z S}^{*}(h)=\frac{1}{2 N(h)} \sum_{|x i-x j|=|h|}\left(z\left(x_{i}\right)-z\left(x_{j}\right)\right)\left(s\left(x_{i}\right)-s\left(x_{j}\right)\right)$

Y se ajusto mediante el modelo de corregionalización lineal con las variables incluidas:

$$
\begin{aligned}
& {\left[\begin{array}{ll}
\gamma_{Z}(h) & \gamma_{Z S}(h) \\
\gamma_{Z S}(h) & \gamma_{S}(h)
\end{array}\right]=\left[\begin{array}{ll}
u_{1} & w_{1} \\
w_{1} & v_{1}
\end{array}\right]\left[\begin{array}{cc}
\gamma_{1}(h) & 0 \\
0 & \gamma_{1}(h)
\end{array}\right]+\Lambda+} \\
& +\left[\begin{array}{cc}
u_{m} & w_{m} \\
w_{m} & v_{m}
\end{array}\right]\left[\begin{array}{cc}
\gamma_{m}(h) & 0 \\
0 & \gamma_{m}(h)
\end{array}\right]
\end{aligned}
$$

Donde $h$ es la distancia entre los pares, $\mathrm{z}$ la variable independiente y $s$ las variables dependientes.

Cokriging es un método multivariable de estimación espacial restringido a una zona de interés, en función de la correlación espacial entre los valores observados a diferentes distancias y direcciones que permite interpolar un valor para los puntos donde no se dispone de información. El método permite explicar una variable en función de otras variables auxiliares correlacionadas espacialmente con ella mediante un semivariograma cruzado ${ }^{17 ; 25}$. Este es el estimador Cokriging ordinario propuesto:

$$
E\left(Z_{c o k}^{*}(u)\right)=m \sum \lambda_{\alpha}+\sum_{j=1}^{K} m_{j} \sum_{\alpha_{j}=1}^{N_{j}} \beta_{\alpha j}
$$

Se calculó el error estándar de todas las estimaciones.
Para la presentación de los resultados se utilizan mapas de distribución de riesgo estimado de TB respiratoria.

Todos los análisis, los gráficos y los mapas han sido realizados con el software libre R v 2.5.1.

\section{RESULTADOS}

En el análisis de los datos se incluyeron 46 de las 47 provincias del territorio peninsular español que disponían de información completa en la RENAVE de casos de TB respiratoria durante el año 2006. Se excluyo una provincia por no estar los datos completos. Los casos totales de TB respiratoria incluidos en el análisis fueron 5.876, siendo el 65\% hombres y el $35 \%$ mujeres. El $65 \%$ tanto en hombres como en mujeres estaban en el rango de edad de 15 a 45 años. Las tasas de incidencia estandarizadas por edad y sexo oscilan entre 10 y 260 por cien mil habitantes existiendo una gran variabilidad espacial.

Siete de las 10 variables incluidas en el modelo GLM resultaron estadísticamente significativas: tasa de hacinamiento, tasa de inmigración, tasa de analfabetismo, tasa de paro, gasto medio en euros por persona/año, tasa de SIDA y tasa de gripe. (Tabla 1).

Para estimar el riesgo de incidencia de TB ajustado por las variables que resultaron estadísticamente significativas se utilizo la técnica de interpolación Cokrigring, mediante un modelo exponencial para ajustar los parámetros de las variables; se utilizó un modelo lineal de corregionalización para obtener las mínimas varianzas del semivariograma cruzado y se calculo el error estándar de las estimaciones.

El patrón de distribución de riesgo de incidencia ajustado por las variables dichas anteriormente, presenta las tasas más altas en la parte noroeste y noreste de la península, así como en el sureste. Los valores mas 
Tabla 1

Asociación de la Tuberculosis con variables socioeconómicas y epidemiológicas.

(Modelo GLM)

\begin{tabular}{|l|c|c|c|c|}
\hline \multicolumn{1}{|c|}{ Tasa TB } & IRR & Error Estándar & \multicolumn{2}{c|}{ Intervalo confianza 95\% } \\
\hline Hacinamiento & 0,945064 & 0,004877 & 0,954673 & 1,9355536 \\
\hline Tasa gripe & 1,000137 & 0,000019 & 1,000098 & 1,000014 \\
\hline Tasa inmigración & 1,000019 & $2,92 \mathrm{E}-06$ & 1,018793 & 1,059743 \\
\hline Tasa SIDA & 1,039066 & 0,010445 & 1,007698 & 1,02093 \\
\hline Analfabetos & 1,014293 & 0,003375 & 0,9513905 & 0,975533 \\
\hline Tasa paro & 0,963386 & 0,006158 & 0,9999437 & 0,9999646 \\
\hline Gasto euros & 0,999954 & $5,34 \mathrm{E}-06$ & & \multicolumn{2}{|c|}{} \\
\hline
\end{tabular}

Figura 1

Riesgo de incidencia de TB ajustado por tasa de hacinamiento, tasa de inmigración, tasa de analfabetismo, tasa de paro, gastos medio en euros por persona/año, tasa de SIDA y tasa de gripe. 2006 y error estándar Tasa de incidencia/100.000

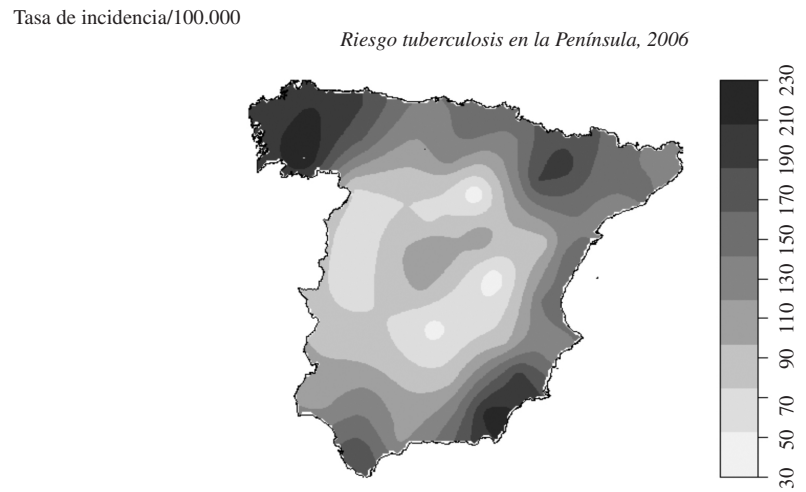

Error Estándar

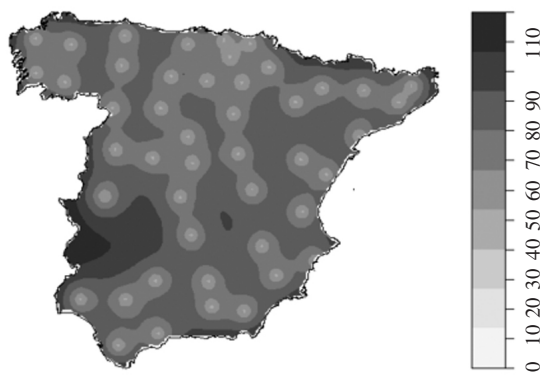

bajos se concentran en el centro, destacando el centro peninsular con un ligero descenso. El error estándar indica menor error en las zonas donde se dispone de información y es más alto a medida que aumenta la distancia. (Figura 2). 


\section{DISCUSIÓN}

Los resultados de nuestro estudio permiten afirmar que el método Cokriging es una herramienta útil para representar la distribución espacial del riesgo de tuberculosis (incidencia) en la península. Mediante el empleo de este método se ha podido estudiar la asociación de la incidencia de tuberculosis con diferentes variables epidemiológicas y socioeconómicas en su distribución geográfica. Los resultados sugieren una asociación entre el riesgo de tuberculosis y la tasa de hacinamiento, tasa de inmigración, tasa de analfabetismo, tasa de paro, gasto medio en euros por persona/año, tasa de SIDA y tasa de gripe.

En España existen estudios que relacionan factores sociales y su distribución por barrios dentro de una ciudad ${ }^{6}$ y otros que relacionan factores epidemiológicos y TB sin tener en cuenta la distribución geográfica ${ }^{15}$.

Este es el primer estudio que analiza la incidencia ajustada por factores socioeconómicos y epidemiológicos y la distribución espacial del riesgo a nivel peninsular, siendo los resultados similares a los obtenidos en otros países y que analizan la asociación de la incidencia de la TB respiratoria con factores socioeconómicos así como su distribución geográfica ${ }^{16 ; 19-21}$.

El patrón de distribución espacial para el riesgo de incidencia de TB es coherente con los datos de la Red Nacional de Vigilancia, siendo las áreas con mayor incidencia las que presentan el riesgo mayor de tuberculosis. Esta distribución no varía sustancialmente al ajustar por las distintas variables del modelo, aunque se suaviza, lo que indica que hay otras variables que influyen en esta distribución y que no se han tenido en cuenta. La tuberculosis se ha asociado tradicionalmente a la deprivación socioeconómica y al hacinamiento, así como a la inmigración de personas procedentes de países de alta endemia $5 ; 6 ; 15 ; 26 ; 27$.
En nuestro estudio se ha encontrado asociación estadísticamente significativa entre el riesgo de tuberculosis respiratoria y la tasa de inmigración, y distintos estudios realizados en España muestran un aumento del porcentaje de inmigrantes respecto al total de casos de tuberculosis en los últimos años $5 ; 26 ; 28$.

Finalmente señalar entre las limitaciones de nuestro estudio que la forma de estimar los riesgos de incidencia no valora el número bruto de casos asociados a la TB respiratoria. La información utilizada tiene carácter poblacional y por lo tanto las asociaciones sugeridas podrían no ser aplicables a nivel individual. La TB respiratoria se trasmite persona a persona lo que influye directamente en la distribución de la enfermedad. Nuestro trabajo no tiene en cuenta los movimientos poblacionales ni los contactos persona a persona. Tampoco se han tenido en cuenta otras variables ambientales o socioeconómicas que podrían influir en la incidencia de tuberculosis.

Nuestro estudio se restringe a las capitales de provincia por no disponer de datos completos en la Red Nacional de Vigilancia desagregados a nivel municipal. La precisión de la distribución de riesgo de TB presentada en los mapas es buena y se corresponde con los datos de la Red Nacional de Vigilancia, sin embargo el número limitado de datos y la distancia entre los centroides de cada provincia conlleva a que en algunas zonas la confianza que se le puede dar a la estimación sea menor como muestran los diferentes mapas de error estándar.

Podemos concluir que la técnica multivariable Cokriging propuesta permite estudiar la asociación de la tuberculosis con diferentes variables controlando por aquellas significativas. Estos métodos geoestadísticos proporcionan un valor añadido a la vigilancia epidemiológica de enfermedades infecciosas y podrían incorporarse de forma rutinaria a la misma. 


\section{BIBLIOGRAFÍA}

1. World Health Organization. Global Tuberculosis Control, 2008. Surveillance, Planning, Financing. WHO/HTM/TB/2008.393.

2. Institut de Veille Sanitaire S-MF. EuroTB and the national coordinators for tuberculosis surveillance in the WHO European Region. Surveillance of tuberculosis in Europe. Report on tuberculosis cases notified in 2006.

3. Allotey P, Gyapong M. Gender in tuberculosis research. Int J Tuberc Lung Dis. 2008; 12: 831-836.

4. Bates M, Khalakdina A, Pai M, Chang L, Lessa F, Smith K. Risk of Tuberculosis From Exposure to Tobacco Smoke. Arch Intern Med. 2007; 167: 335-342.

5. Ordovás $\mathrm{M}$, Cañellas $\mathrm{M}$, García C, García L, Gutierrez M, Rodero I et al. Tuberculosis en la Comunidad de Madrid. Incidencia en personas extranjeras y españolas durante el periodo 19962004. Rev Esp Salud Pública 2007; 81: 597-604.

6. Díaz de Quijano E, Brugal M, Pasarín M, GaldósTangüis H, Caylá J, Borrell C. Influencia de las desigualdades sociales, la conflictividad social y la pobreza extrema sobre la morbilidad por tuberculosis en la ciudad de Barcelona. Rev Esp Salud Pública 2001; 75: 517-528.

7. Kistemann T, Munzinger A, Dangendorf F. Spatial patterns of tuberculosis incidence in Cologne (Germany). Soc Sci Med. 2002; 55: 7-19.

8. Real Decreto 2210/1995 por el que se crea la Red Nacional de Vigilancia Epidemiológica. Boletín Oficial Del Estado núm de 29/12/1995.

9. Ministerio de Sanidad y Consumo. Protocolos de las Enfermedades de Declaración Obligatoria. Madrid: Ministerio de Sanidad y Consumo; 2001.

10. Ampliación de la definición de caso de tuberculosis en la Red Nacional de Vigilancia Epidemiológica (I). Bol Epidemiol Sem. 2003; 11: 181-184.

11. Ampliación de la definición de caso de tuberculosis en la Red Nacional de Vigilancia Epidemiológica (II). Bol Epidemiol Sem 2003; 11: 193-195.

12. Registro Nacional de Sida. Vigilancia Epidemiológica del sida en España. Situación a 30 de junio de 2006. Bol Epidemiol Sem. 2006; 14: 133-136.

13. Rodríguez E, Díaz O, Hernández M, Hernández G, Tello O. Casos de tuberculosis declarados a la
Red Nacional de Vigilancia Epidemiológica. España, 2005-2006. Bol Epidemiol Sem. 2007; 15: 205-208.

14. Grupo de Trabajo del PMIT. La tuberculosis en España: resultados del Proyecto Multicéntrico de Investigación sobre Tuberculosis (PMIT). Madrid:Instituto de Salud Carlos III;1999.

15. Diez M, Diaz A, Bleda MJ, Aldamiz M, Camafort M, Camino X et al. Prevalence of M. tuberculosis infection and tuberculosis disease among HIVinfected people in Spain. Int J Tuberc Lung Dis. 2007; 11: 1196-1202.

16. Chan-yeung M, Yeh AG, Tam CM, Kam KM, Leung CC, Yew WW et al. Socio-demographic and geographic indicators and distribution of tuberculosis in Hong Kong: a spatial analysis. Int J Tuberc Lung Dis. 2005; 9: 1320-1326.

17. Valencia LI, Fortes BP, Medronho RA. Spatial ascariasis risk estimation using socioeconomic variables. Int J Environ.Health Res. 2005; 15: 411-424.

18. Egunjobi L. Spatial distribution of mortality from leading notifiable diseases in Nigeria. Soc Sci Med. 1993; 36: 1267-1272.

19. Mota FF, Vieira-da-Silva LM, Paim JS, Costa MC. Spatial distribution of tuberculosis mortality in Salvador, Bahia, Brazil. Cad.Saude Publica. 2003; 19: 915-922.

20. Rodrigues AL, Jr., Ruffino-Netto A, de Castilho EA. Spatial distribution of M. tuberculosis-HIV coinfection in Sao Paulo State, Brazil, 1991-2001. Rev Saude Publica. 2006; 40: 265-270.

21. Vieira RC, Prado TN, Siqueira MG, Dietze R, Maciel EL. Spatial distribution of new tuberculosis cases in Vitoria, State of Espirito Santo, between 2000 and 2005. Rev Soc Bras.Med Trop. 2008; 41: 82-86.

22. McCullagh and Neder. Generalizer Linear Model,GLM. London: Chapman and Hall; 1989.

23. Estrada-Pena A. Geostatistics and remote sensing as predictive tools of tick distribution: a cokriging system to estimate Ixodes scapularis (Acari: Ixodidae) habitat suitability in the United States and Canada from advanced very high resolution radiometer satellite imagery. J Med Entomol. 1998; 35: 989-995.

24. Giraldo R. Propuesta de un indicador como variable auxiliar en el analisis cokriging. Rev Colombiana Estadist 2001; 24: 1-12. 
25. Goovaerts P. Ordinary Cokriging Revisited. Available: http://hdl.handle.net/2027.42/43198

26. Chaves F, Iñigo J. Tuberculosis e inmigración en España. Rev Esp Salud Pública 2007; 81: 585587.

27. Lefebvre N, Falzon D. Risk factors for death among tuberculosis cases: analysis of European surveillance data. Eur Respir.J. 2008; 31: 12561260 .

28. Generalitat de Catalunya.Situación epidemiológica y tendencia de la endemia tuberculosa en Cataluña. Informe anual 2006.Departament de Salut. Generalitat de Catalunya. Programa de Prevenció i Control de la Tuberculosi. Generalitat de Catalunya;2008. 